\title{
Power Minimization in Wireless Sensor Networks With Constrained AoI Using Stochastic Optimization
}

\author{
Mohammad Moltafet and Markus Leinonen \\ Centre for Wireless Communications - Radio Technologies \\ University of Oulu, Finland \\ e-mail: \{mohammad.moltafet, markus.leinonen\}@oulu.fi
}

\author{
Marian Codreanu and Nikolaos Pappas \\ Department of Science and Technology \\ Linköping University, Sweden \\ e-mail: \{marian.codreanu, nikolaos.pappas\}@liu.se
}

\begin{abstract}
In this paper, we consider a system where multiple low-power sensors communicate timely information about a random process to a sink. The sensors share orthogonal subchannels to transmit such information in the form of status update packets. Freshness of the sensors' information at the sink is characterized by the Age of Information (AoI), and the sensors can control the sampling policy by deciding whether to take a sample or not. We formulate an optimization problem to minimize the time average total transmit power of sensors by jointly optimizing the sampling action of each sensor, the transmit power allocation, and the subchannel assignment under the constraints on the maximum time average AoI and maximum power of each sensor. To solve the optimization problem, we use the Lyapunov drift-plus-penalty method. Numerical results show the performance of the proposed algorithm versus the different parameters of the system.

Index Terms- Age of Information (AoI), Information freshness, Lyapunov optimization, power minimization.
\end{abstract}

\section{INTRODUCTION}

Freshness of the status information of various physical processes collected by multiple sensors is a key performance enabler in many applications of wireless sensor networks (WSNs) [1]-[3], e.g., surveillance in smart home systems and drone control. The Age of Information (AoI) was introduced as a destination centric metric that characterizes the freshness in status update systems [4]-[6]. A status update packet of each sensor contains a time stamp representing the time when the sample was generated and the measured value of the monitored process. If at a time instant $t$, the most recently received status update packet contains the time stamp $U(t)$, the AoI is defined as $\Delta(t)=t-U(t)$. In other words, the AoI of each sensor is the time elapsed since the last received status update packet was generated at the sensor. The average AoI is the most commonly used metric to evaluate the AoI [2]-[12].

The authors of [7] considered a WSN in which sensors share one unreliable subchannel in each slot. They minimized the expected weighted sum AoI of the network by determining the transmission scheduling policy. The authors of [8] considered an energy harvesting sensor and derived the optimal threshold in terms of remaining energy to trigger a new sample. The authors of [11] considered an energy harvesting sensor and minimized the time average AoI by determining the optimal status update policy. The authors of [9] considered two source nodes generating heterogeneous traffic with different power supplies and studied the peak-age-optimal status update scheduling. The authors of [10] considered a wireless power transfer powered sensor network and studied performance of the system in terms of the average AoI.

In this paper, we minimize the time average total transmit power of sensors by jointly optimizing the sampling action, the transmit power allocation, and the subchannel assignment in each slot under the constraints on the maximum time average AoI and maximum power of each sensor. To solve the proposed optimization problem, we apply the Lyapunov drift-plus-penalty method. To the best of our knowledge, joint optimization of the transmit power allocation, subchannel assignment, and sampling action with constrained AoI has not been studied earlier. The most related work to this paper is [7]. Differently from [7], besides the sampling action of each sensor, we consider both transmit power allocation and subchannel assignment in each slot.

\section{System Model and Problem Formulation}

We consider a WSN consisting of a set $\mathcal{K}$ of $K$ sensors and one sink, as depicted in Fig. 1. The sink is interested in time-sensitive information from the sensors which measure a physical phenomenon. We assume a slotted communication with normalized slots $t \in\{1,2, \ldots\}$, where in each slot, the sensors share a set $\mathcal{N}$ of $N$ orthogonal subchannels with bandwidth $W$ per subchannel. We consider that each sensor can control the sampling process by deciding whether to take a sample or not at the beginning of each slot $t$. We assume that the perfect channel state information is available at the sink.

Let $\rho_{k, n}(t)$ denote the subchannel assignment at time slot $t$ as $\rho_{k, n}(t) \in\{0,1\}, \forall k \in \mathcal{K}, n \in \mathcal{N}$, where $\rho_{k, n}(t)=1$ indicates that subchannel $n$ is assigned to sensor $k$ at time slot $t$, and $\rho_{k, n}(t)=0$ otherwise. To ensure that at any given time slot $t$, each subchannel can be assigned to at most one sensor, the following constraint is used:

$$
\sum_{k \in \mathcal{K}} \rho_{k, n}(t) \leq 1, n \in \mathcal{N}, \forall t .
$$




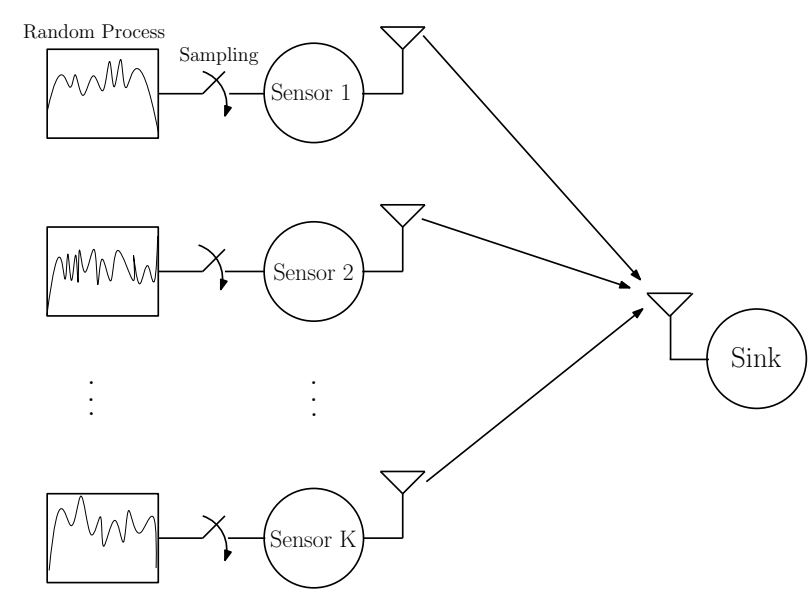

Fig. 1: System model.

Let $p_{k, n}(t)$ denote the transmitted power of sensor $k$ over subchannel $n$ at slot $t$. Then, the signal-to-noise ratio (SNR) with respect to sensor $k$ over subchannel $n$ at slot $t$ is given by

$$
\gamma_{k, n}(t)=\frac{p_{k, n}(t)\left|h_{k, n}(t)\right|^{2}}{W N_{0}},
$$

where $h_{k, n}(t)$ is the channel coefficient from sensor $k$ to the sink over subchannel $n$ at slot $t$ and $N_{0}$ is the noise power spectral density. Accordingly, the achievable rate for sensor $k$ over subchannel $n$ in slot $t$ is given by

$$
r_{k, n}(t)=W \log _{2}\left(1+\gamma_{k, n}(t)\right) .
$$

The achievable data rate of sensor $k$ at slot $t$ is equal to the summation of achievable data rates over all assigned subchannels at slot $t$, expressed as

$$
R_{k}(t)=\sum_{n \in \mathcal{N}} \rho_{k, n}(t) r_{k, n}(t) .
$$

Let $b_{k}(t)$ denote the sampling action of sensor $k$ at time slot $t$ as $b_{k}(t) \in\{0,1\}, \forall k \in \mathcal{K}$, where $b_{k}(t)=1$ indicates that sensor $k$ takes a sample at the beginning of time slot $t$, and $b_{k}(t)=0$ otherwise. We assume that sampling time (i.e., the time needed to take a sample) is negligible. We consider that sensor $k$ takes a sample at the beginning of slot $t$ only if there are enough resources to transmit the sample during the same slot $t$. In other words, if sensor $k$ takes a sample at the beginning of slot $t$ (i.e., $b_{k}(t)=1$ ), the sample will be transmitted during the same slot $t$. To this end, we use the following constraint:

$$
R_{k}(t)=\eta b_{k}(t), k \in \mathcal{K}, \forall t,
$$

where $\eta$ is the size of each status update packet (bits). This constraint ensures that when sensor $k$ takes a sample at the beginning of slot $t$ (i.e., $b_{k}(t)=1$ ), the achievable rate for sensor $k$ at slot $t$ is $R_{k}(t)=\eta$, which guarantees that the sample is transmitted during the slot.

Let $\delta_{k}(t)$ denote the AoI of the sensor $k$ at the beginning of slot $t$. If sensor $k$ takes a sample at the beginning of slot $t$ (i.e., $b_{k}(t)=1$ ), the AoI at the beginning of slot $t+1$ drops

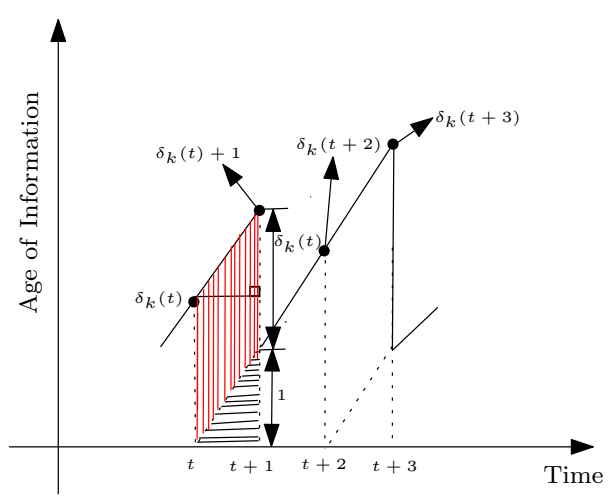

Fig. 2: The evolution of the AoI of sensor $k$.

to one, and otherwise (i.e., $b_{k}(t)=0$ ), the AoI is incremented by one. Accordingly, the evolution of $\delta_{k}(t)$ is characterized as

$$
\delta_{k}(t+1)= \begin{cases}1 & , \text { if } b_{k}(t)=1 ; \\ \delta_{k}(t)+1 & , \text { otherwise }\end{cases}
$$

The evolution of the AoI of sensor $k$ is illustrated in Fig. 2. The time average AoI of sensor $k$ is calculated as the area under the AoI curve, normalized by the observation interval. As it can be seen, during slot $t$, the area under the AoI curve of sensor $k$ is calculated as a sum of the areas of a triangle and a parallelogram. The area of the triangle is equal to $1 / 2$ and the area of the parallelogram is equal to $\delta_{k}(t)$. Therefore, the time average AoI of sensor $k$ is calculated as

$$
\Delta_{k}=\frac{1}{2}+\lim _{t \rightarrow \infty} \frac{1}{t} \sum_{\tau=1}^{t} \delta_{k}(\tau) .
$$

To make the calculations tractable, we use a commonly used approach that instead of the time average AoI in (6), we consider the time average of expectation of the AoI [7], [13], [14], given as

$$
\Delta_{k}=\frac{1}{2}+\lim _{t \rightarrow \infty} \frac{1}{t} \sum_{\tau=1}^{t} \mathbb{E}\left[\delta_{k}(\tau)\right],
$$

where the expectation is with respect to the random wireless channel states and control actions made in reaction to the channel states ${ }^{1}$. We consider that the initial value of the AoI of all sensors is $\delta_{k}(1)=0, \forall k \in \mathcal{K}$.

\section{A. Problem Formulation}

Our objective is to minimize the time average total transmit power of sensors by optimizing the sampling action, the transmit power allocation, and the subchannel assignment in each slot subject to the maximum time average AoI and maximum power constraints for each sensor. Thus, the optimization problem is formulated as follows

$$
\begin{array}{ll}
\text { minimize } & \lim _{t \rightarrow \infty} \frac{1}{t} \sum_{\tau=1}^{t} \sum_{k \in \mathcal{K}} \sum_{n \in \mathcal{N}} \mathbb{E}\left[p_{k, n}(\tau)\right] \\
\text { subject to } \quad \sum_{k \in \mathcal{K}} \rho_{k, n}(t) \leq 1, \forall n \in \mathcal{N}, \forall t
\end{array}
$$

\footnotetext{
${ }^{1}$ Through the paper, all expectations are taken with respect to the randomness of the wireless channel states and control actions made in reaction to the channel states.
} 


$$
\begin{aligned}
& \sum_{n \in \mathcal{N}} p_{k, n}(t) \leq P_{k}^{\max }, \quad k \in \mathcal{K}, \forall t \\
& \Delta_{k} \leq \Delta_{k}^{\max }, \quad k \in \mathcal{K} \\
& \sum_{n \in \mathcal{N}} \rho_{k, n}(t) r_{k, n}(t)=\eta b_{k}(t), k \in \mathcal{K}, \forall t \\
& \rho_{k, n}(t) \in\{0,1\}, k \in \mathcal{K}, n \in \mathcal{N}, \forall t \\
& b_{k}(t) \in\{0,1\}, k \in \mathcal{K}, \forall t
\end{aligned}
$$

with variables $\left\{p_{k, n}(t), \rho_{k, n}(t)\right\}_{k \in \mathcal{K}, n \in \mathcal{N}}$ and $\left\{b_{k}(t)\right\}_{k \in \mathcal{K}}$ for all $t \in\{1,2, \ldots\}$. The constraints of problem (8) are as follows. The inequality ( $8 b)$ constrains that each subchannel can be assigned to at most one sensor in each slot; the inequality (8c) constrains the power of each sensor with respect to the maximum budget $P_{k}^{\max }$; the inequality (8d) is the maximum acceptable time average AoI constraint for each sensor; the equality (8e) ensures that each sample is transmitted during one slot; (8f) and (8g) represent the feasible values for the subchannel assignment and sampling policy variables, respectively.

The proposed optimization problem is a mixed integer programming problem where the constraints and the objective function both contain time averages over the optimization variables. In the following section, a dynamic control algorithm using the Lyapunov optimization approach is presented to solve optimization problem (8).

\section{Solution Algorithm}

We use the Lyapunov drift-plus-penalty method introduced in [13] and [14] to solve the optimization problem (8). According to the drift-plus-penalty method, the time average constraint (8d) is enforced by transforming the problem into a queue stability problem. In other words, for each time average inequality in constraint (8d) a virtual queue is associated in such a way that the stability of these virtual queues implies the feasibility of constraint (8d).

To use the drift-plus penalty method, we rewrite constraint (8d) as follows

$$
\lim _{t \rightarrow \infty} \frac{1}{t} \sum_{\tau=1}^{t} \mathbb{E}\left[\delta_{k}(\tau)\right] \leq \Delta_{k}^{\max }-\frac{1}{2}, \quad k \in \mathcal{K} .
$$

Let $\left\{Q_{k}(t)\right\}_{k \in \mathcal{K}}$ denote the virtual queues associated with constraint (9). Then, the virtual queues are updated at the beginning of each time slot as

$Q_{k}(t+1)=\max \left[Q_{k}(t)-\left(\Delta_{k}^{\max }-\frac{1}{2}\right), 0\right]+\delta_{k}(t+1), \forall k \in \mathcal{K}$.

Here, we use the notion of strong stability; the virtual queues are strongly stable if [13, Ch. 2]

$$
\lim _{t \rightarrow \infty} \frac{1}{t} \sum_{\tau=1}^{t} \mathbb{E}\left[Q_{k}(\tau)\right]<\infty, \forall k \in \mathcal{K}
$$

According to (11), a queue is strongly stable if its time average backlog is finite. Next, we introduce the Lyapunov function and its drift which are needed to define the queue stability problem.

Let $\mathbf{S}(t)=\left\{Q_{k}(t), \delta_{k}(t)\right\}_{k \in \mathcal{K}}$ denote the network state at the beginning of slot $t$, and $\mathbf{Q}(t)$ denote a vector containing all the virtual queues, i.e., $\mathbf{Q}(t)=\left[Q_{1}(t), Q_{2}(t), \ldots, Q_{K}(t)\right]$. Then, a quadratic Lyapunov function $L(\mathbf{Q}(t))$ is defined by [13, Ch. 3]

$$
L(\mathbf{Q}(t))=\frac{1}{2} \sum_{k \in \mathcal{K}} Q_{k}^{2}(t) .
$$

The Lyapunov function measures the network congestion: if the Lyapunov function is small, then all the queues are small, and if the Lyapunov function is large, then at least one queue is large. Therefore, by minimizing the expected change of the Lyapunov function from one slot to the next slot, queues $\left\{Q_{k}(t)\right\}_{k \in \mathcal{K}}$ can be stabilized [13, Ch. 4].

The expected of the Lyapunov function from one slot to the next slot is defined as the drift in the Lyapunov function, which is defined as

$$
\alpha(\mathbf{S}(t))=\mathbb{E}[L(\mathbf{Q}(t+1))-L(\mathbf{Q}(t)) \mid \mathbf{S}(t)] .
$$

According to the drift-plus-penalty minimization method, a control policy that minimizes the objective function of the optimization problem (8) is obtained by minimizing the driftplus-penalty in each slot $t[13$, Ch. 3], i.e.,

$$
\alpha(\mathbf{S}(t))+V \sum_{k \in \mathcal{K}} \sum_{n \in \mathcal{N}} \mathbb{E}\left[p_{k, n}(t)\right],
$$

subject to the following constraints

$$
\begin{aligned}
& \sum_{k \in \mathcal{K}} \rho_{k, n}(t) \leq 1, \forall n \in \mathcal{N}, \forall t \\
& \sum_{n \in \mathcal{N}} p_{k, n}(t) \leq P_{k}^{\max }, \quad k \in \mathcal{K}, \forall t \\
& R_{k}(t)=\eta b_{k}(t), k \in \mathcal{K}, \forall t \\
& \rho_{k, n}(t) \in\{0,1\}, k \in \mathcal{K}, n \in \mathcal{N}, \forall t \\
& b_{k}(t) \in\{0,1\}, k \in \mathcal{K}, \forall t,
\end{aligned}
$$

where $V \geq 0$ is a parameter that represents how much we emphasize on the objective function (power minimization). Therefore, by varying $V$, a desired trade-off between the sizes of the queue backlogs and objective function can be obtained.

Since minimizing the objective function (14) is intractable, we minimize an upper bound of (14) in each slot $t[13, \mathrm{Ch}$. 3]. To find an upper bound for (14), we use the following inequality in which for any $\hat{A} \geq 0, \tilde{A} \geq 0$, and $\bar{A} \geq 0$ we have [13, Ch. 3]

$$
(\max [\hat{A}-\tilde{A}, 0]+\bar{A})^{2} \leq \hat{A}^{2}+\tilde{A}^{2}+\bar{A}^{2}+2 \hat{A}(\bar{A}-\tilde{A}) .
$$

By applying (16) to (10), an upper bound for $Q_{k}^{2}(t+1)$ is given as

$$
\begin{aligned}
& Q_{k}^{2}(t+1) \leq Q_{k}^{2}(t)+\left(\Delta_{k}^{\max }-\frac{1}{2}\right)^{2}+\delta_{k}^{2}(t+1)+2 Q_{k}(t) \\
& \left(\delta_{k}(t+1)-\left(\Delta_{k}^{\max }-\frac{1}{2}\right)\right) .
\end{aligned}
$$

By applying (17) to (14), an upper bound for (14) is given as

$$
\begin{aligned}
& \alpha(\mathbf{S}(t))+V \sum_{k \in \mathcal{K}} \sum_{n \in \mathcal{N}} \mathbb{E}\left[p_{k, n}(t)\right] \leq V \sum_{k \in \mathcal{K}} \sum_{n \in \mathcal{N}} \mathbb{E}\left[p_{k, n}(t)\right]+ \\
& \frac{1}{2} \mathbb{E}\left[\sum _ { k \in \mathcal { K } } \left(\left(\Delta_{k}^{\max }-\frac{1}{2}\right)^{2}+\delta_{k}^{2}(t+1)+2 Q_{k}(t)\left(\delta_{k}(t+1)-\right.\right.\right.
\end{aligned}
$$




$$
\begin{aligned}
& \left.\left.\left.\left(\Delta_{k}^{\max }-\frac{1}{2}\right)\right)\right) \mid \mathbf{S}(t)\right]=V \sum_{k \in \mathcal{K}} \sum_{n \in \mathcal{N}} \mathbb{E}\left[p_{k, n}(t)\right] \\
& +\frac{1}{2} \sum_{k \in \mathcal{K}}\left(\left(\Delta_{k}^{\max }-\frac{1}{2}\right)^{2}+\mathbb{E}\left[\delta_{k}^{2}(t+1) \mid \mathbf{S}(t)\right]+2 Q_{k}(t)\right. \\
& \left.\left(\mathbb{E}\left[\delta_{k}(t+1) \mid \mathbf{S}(t)\right]-\left(\Delta_{k}^{\max }-\frac{1}{2}\right)\right)\right) .
\end{aligned}
$$

To derive the upper bound for (14), we need to determine $\mathbb{E}\left[\delta_{k}(t+1) \mid \mathbf{S}(t)\right]$ and $\mathbb{E}\left[\delta_{k}^{2}(t+1) \mid \mathbf{S}(t)\right]$. To this end, by using the evolution of the AoI in (5), $\delta_{k}(t+1)$ and $\delta_{k}^{2}(t+1)$ are calculated as

$$
\begin{aligned}
& \delta_{k}(t+1)=b_{k}(t)+\left(1-b_{k}(t)\right)\left(\delta_{k}(t)+1\right), k \in \mathcal{K} \\
& \delta_{k}^{2}(t+1)=b_{k}(t)+\left(1-b_{k}(t)\right)\left(\delta_{k}(t)+1\right)^{2}, k \in \mathcal{K} .
\end{aligned}
$$

By using the expressions in (19), $\mathbb{E}\left[\delta_{k}(t+1) \mid \mathbf{S}(t)\right]$ and $\mathbb{E}\left[\delta_{k}^{2}(t+1) \mid \mathbf{S}(t)\right]$ are given as

$\mathbb{E}\left[\delta_{k}(t+1) \mid \mathbf{S}(t)\right]=\mathbb{E}\left[b_{k}(t)\right]+\left(1-\mathbb{E}\left[b_{k}(t)\right]\right)\left(\delta_{k}(t)+1\right), k \in \mathcal{K}$

$\mathbb{E}\left[\delta_{k}^{2}(t+1) \mid \mathbf{S}(t)\right]=\mathbb{E}\left[b_{k}(t)\right]+\left(1-\mathbb{E}\left[b_{k}(t)\right]\right)\left(\delta_{k}(t)+1\right)^{2}, k \in \mathcal{K}$.

By substituting (20) into the right hand side of (18), the upper bound for (14) is given as

$$
\begin{aligned}
& V \sum_{k \in \mathcal{K}} \sum_{n \in \mathcal{N}} \mathbb{E}\left[p_{k, n}(t)\right]+\frac{1}{2} \sum_{k \in \mathcal{K}}\left(\left(\Delta_{k}^{\max }-\frac{1}{2}\right)^{2}+\left(\delta_{k}(t)+1\right)^{2}\right. \\
& +\left(2 Q_{k}(t)-1\right)\left(\delta_{k}(t)+1\right)+\mathbb{E}\left[b_{k}(t)\right]\left(1-\left(\delta_{k}(t)+1\right)^{2}\right. \\
& \left.\left.-2 Q_{k}(t) \delta_{k}(t)\right)\right) .
\end{aligned}
$$

Next, we explain the proposed dynamic algorithm to solve the optimization problem (8). The main steps of the algorithm are summarized in Algorithm 1. The algorithm observes the channel states $\left\{h_{k, n}(t)\right\}_{k \in \mathcal{K}, n \in \mathcal{N}}$ and network state $\mathbf{S}(t)$ in each time slot $t$ and makes a control action to minimize (21) subject to the constraints (15a)-(15e). Note that the driftplus penalty method exploits the opportunistically minimize an expectation [13, Ch. 8] to solve the subproblem in each slot. To solve the optimization problem (22) in each slot, we confine to use the exhaustive search algorithm.

\section{Numerical Results}

In this section, we evaluate the performance of the proposed dynamic control algorithm presented in Algorithm 1. Due to the complexity of the exhaustive search solution used to solve the optimization problem (22), we evaluate the performance of the system with a small number of sensors and subchannels. We consider $K=2$ sensors placed in a two-dimensional plane and $N=2$ subchannels with bandwidth $W=180 \mathrm{kHz}$. The coordinate of sensor 1 is $(0,300)$, the coordinate of sensor 2 is $(300,0)$, and the coordinate of the sink is $(0,0)$. The channel coefficient from sensor $k$ to the sink over subchannel $n$ at slot $t$ is modeled by $h_{k, n}(t)=\left(d_{k} / d_{0}\right)^{\xi} c_{k, n}(t)$, where $d_{k}$ is the distance from sensor $k$ to the sink, $d_{0}$ is the far field reference distance, $\xi$ is the path loss exponent, and $c_{k, n}(t)$ is a Rayleigh distributed random coefficient. Accordingly, $\left(d_{k} / d_{0}\right)^{\xi}$ represents large scale fading and the term $c_{k, n}(t)$
Algorithm 1 Proposed solution algorithm for problem (8)

Step 1: initialization: set $t=0$, set $V$, and initialize $\left\{Q_{k}(0), \delta_{k}(0)\right\}_{k \in \mathcal{K}}$,

for each time slot $t$ do

Step 2: Sampling action, transmit power, and subchannel assignment: obtain $\left\{p_{k, n}(t), \rho_{k, n}(t)\right\}_{k \in \mathcal{K}, n \in \mathcal{N}}$ and $\left\{b_{k}(t)\right\}_{k \in \mathcal{K}}$ by solving the following optimization problem:

$$
\begin{aligned}
\operatorname{minimize} & V \sum_{k \in \mathcal{K}} \sum_{n \in \mathcal{N}} p_{k, n}(t)+ \\
& \frac{1}{2} \sum_{k \in \mathcal{K}}\left(b_{k}(t)\left(1-\left(\delta_{k}(t)+1\right)^{2}-2 Q_{k}(t) \delta_{k}(t)\right)\right)
\end{aligned}
$$

subject to $(15 \mathrm{a})-(15 \mathrm{e})$,

with variables $\quad\left\{p_{k, n}(t), \rho_{k, n}(t)\right\}_{k \in \mathcal{K}, n \in \mathcal{N}} \quad$ and $\left\{b_{k}(t)\right\}_{k \in \mathcal{K}}$

Step 3: Queue update: update $\left\{Q_{k}(t+1), \delta_{k}(t+1)\right\}_{k \in \mathcal{K}}$ using (10) and (19),

Set $t=t+1$, and go to Step 2,

end for

denotes small scale Rayleigh fading. We set $\xi=-3, d_{0}=1$, maximum acceptable average AoI of sensors $\Delta_{k}^{\max }=4, \forall k$, the size of each packet $\eta=600$ Bytes, and the parameter of Rayleigh distribution is 0.5 .

Fig. 3 depicts the average AoI of sensor 1 as a function of $V$. According to this figure, when $V$ increases, the average AoI of sensor 1 increases as well. This is because when $V$ increases, the backlogs of the virtual queues associated to the time average AoI constraints (8d) increase. We can also observe that the average AoI of the sensor is always smaller than the maximum acceptable average AoI $\Delta_{k}^{\max }=4$.

Fig. 4 illustrates the time average total transmit power as a function of $V$. The figure shows that when $V$ increases, the average total transmit power decreases. This is because when $V$ increases, more emphasis is set to minimize the total transmit power in the objective function of optimization problem (22).

Fig. 5 illustrates the trade-off between the average AoI and average total transmit power of the sensors for different values of $V$. By increasing $V$ the average AoI of different sensors increases and the average total transmit power decreases. Note that the average AoI of sensors remains always smaller than the maximum acceptable average AoI.

From Figs. 3 and 5, we observe that by increasing $V$ sufficiently high, the AoI values of the sensors eventually reach the maximum acceptable average AoI. Similarly, as it can be seen in Figs. 4 and 5, for the high values of $V$, the average total transmit power of the sensors starts to saturate into a certain level.

\section{CONCLUSiOnS}

In this paper, we considered a status update system consisting of a set of sensors that can control the sampling action. 


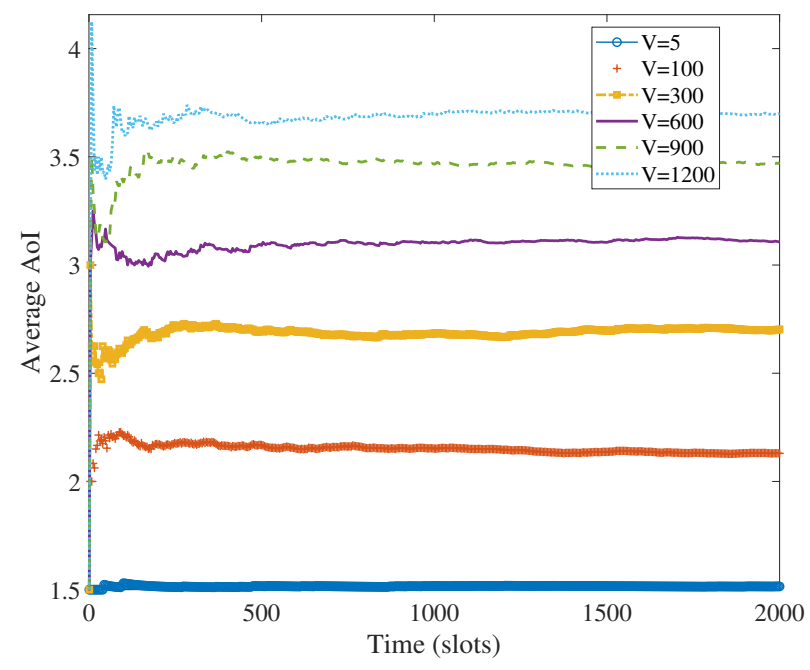

Fig. 3: Average AoI of sensor 1 as a function of $V$.

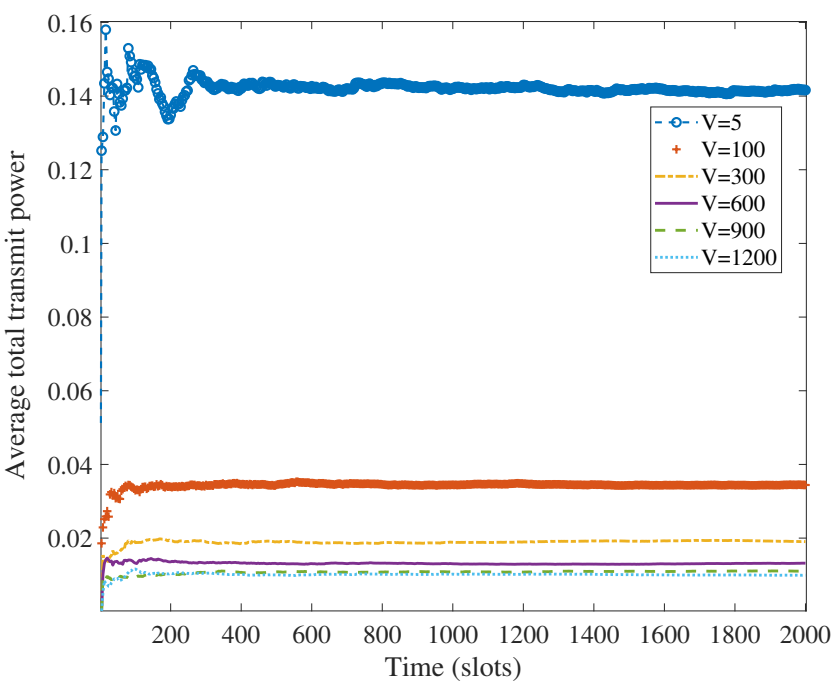

Fig. 4: Average total transmit power of the sensors as a function of $V$.

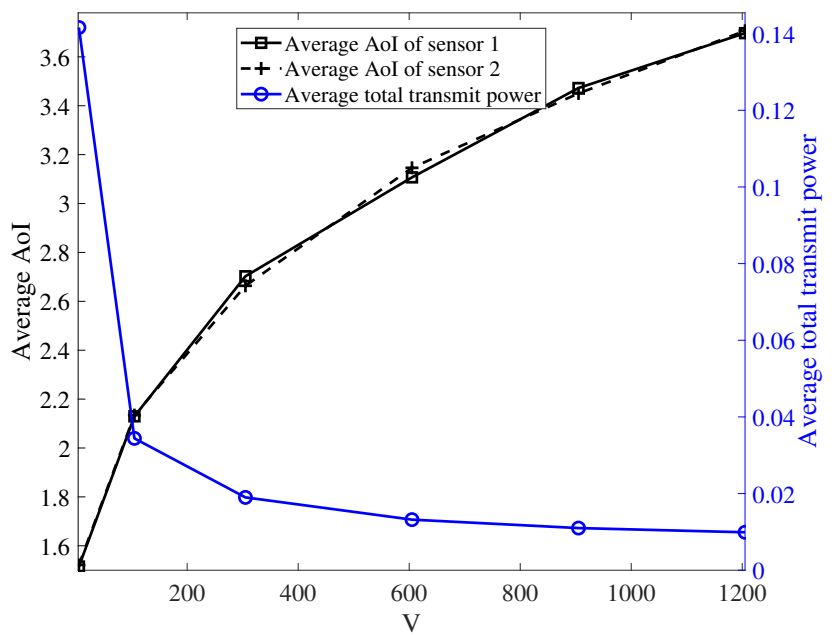

Fig. 5: Trade-off between the average total transmit power and average AoI of sensor 1 and sensor 2 as a function of $V$.
The status update packets of the sensors are transmitted by sharing a set of orthogonal subchannels in each slot. We formulated an optimization problem to minimize the time average total transmit power of sensors with time average AoI and maximum power constraints for each sensor. To solve the proposed optimization problem, we used the Lyapunov drift-plus-penalty method. This method provides a trade-off between the average total transmit power and the average AoI of the sensors which were shown in the numerical experiments.

\section{ACKNOWLEDGEMENTS}

This research has been financially supported by the Infotech Oulu, the Academy of Finland (grant 323698), and Academy of Finland 6Genesis Flagship (grant 318927). M. Codreanu would like to acknowledge the support of the European Union's Horizon 2020 research and innovation programme under the Marie Skłodowska-Curie Grant Agreement No. 793402 (COMPRESS NETS). M. Moltafet would like to acknowledge the support of Finnish Foundation for Technology Promotion.

\section{REFERENCES}

[1] A. Kosta, N. Pappas, and V. Angelakis, "Age of information: A new concept, metric, and tool," Foun. and Trends in Net., vol. 12, no. 3, pp. 162-259, 2017.

[2] R. D. Yates and S. K. Kaul, "The age of information: Real-time status updating by multiple sources," IEEE Trans. Inform. Theory, vol. 65, no. 3, pp. 1807-1827, Mar. 2019.

[3] M. Moltafet, M. Leinonen, and M. Codreanu, "Worst case age of information in wireless sensor networks: A multi-access channel," IEEE Wireless Commun. Lett., Early Access 2019.

[4] S. Kaul, R. Yates, and M. Gruteser, "Real-time status: How often should one update?" in Proc. IEEE Int. Conf. on Computer. Commun. (INFOCOM), Orlando, FL, USA, Mar. 25-30, 2012, pp. 2731-2735.

[5] S. K. Kaul, R. D. Yates, and M. Gruteser, "Status updates through queues," in Proc. Conf. Inform. Sciences Syst. (CISS), Princeton, NJ, USA, Mar. 21-23, 2012, pp. 1-6.

[6] S. Kaul, M. Gruteser, V. Rai, and J. Kenney, "Minimizing age of information in vehicular networks," in Proc. Commun. Society. Conf. on Sensor, Mesh and Ad Hoc Commun. and Net., Salt Lake City, UT, USA, Jun. 27-30, 2011, pp. 350-358.

[7] I. Kadota, A. Sinha, and E. Modiano, "Optimizing age of information in wireless networks with throughput constraints," in Proc. IEEE Int. Conf. on Computer. Commun. (INFOCOM), Honolulu, HI, USA, Apr. 15-19, 2018, pp. 1844-1852.

[8] B. T. Bacinoglu and E. Uysal-Biyikoglu, "Scheduling status updates to minimize age of information with an energy harvesting sensor," in Proc. IEEE Int. Symp. Inform. Theory, Aachen, Germany, Jun. 25-30, 2017.

[9] Z. Chen, N. Pappas, E. Björnson, and E. G. Larsson, "Optimal control of status updates in a multiple access channel with stability constraints," 2019, https://arxiv.org/abs/1910.05144.

[10] I. Krikidis, "Average age of information in wireless powered sensor networks," IEEE Wireless Commun. Lett., vol. 8, no. 2, pp. 628-631, Apr. 2019.

[11] X. Wu, J. Yang, and J. Wu, "Optimal status update for age of information minimization with an energy harvesting source," IEEE Trans. Green Comm. Net., vol. 2, no. 1, pp. 193-204, Mar. 2018.

[12] M. Moltafet, M. Leinonen, and M. Codreanu, "On the age of information in multi-source queueing models," [Online]. https://arxiv.org/abs/1911.07029v1, 2019.

[13] M. J. Neely, Stochastic network optimization with application to communication and queueing systems. Belmont, MA, USA: Morgan and Claypool, 2010.

[14] L. Georgiadis, M. J. Neely, and L. Tassiulas, "Resource allocation and cross-layer control in wireless networks," Found. Trends Netw., vol. 1, no. 1, pp. 1-144, Apr. 2006. 